\title{
A Golden Gate-based Plasmid Library for the Rapid Assembly of Biotin
}

\section{Ligase Constructs for Proximity Labelling}

Kevin Goslin, Andrea Finocchio, and Frank Wellmer*

Smurfit Institute of Genetics, Trinity College Dublin, Ireland

Corresponding author: wellmerf@tcd.ie,+353-1-8963729

Running title: A plasmid library for proximity labelling

Keywords: proximity labelling, biotin ligase, BioID, TurboID, Golden Gate cloning 


\begin{abstract}
Proximity-labelling has emerged as a powerful tool for the detection of weak and transient interactions between proteins as well as the characterization of subcellular proteomes. One proximity labelling approach makes use of a promiscuous bacterial biotin ligase, termed BioID. Expression of BioID (or of its derivates TurboID and MiniTurbo) fused to a bait protein results in the biotinylation of proximal proteins. These biotinylated proteins can then be isolated by affinity purification using streptavidin-coated beads and identified by mass spectrometry. To facilitate the use of proximity-labelling in plants, we have generated a collection of constructs that can be used for the rapid cloning of TurboID and MiniTurbo fusion proteins using the Golden Gate cloning method. To allow for the use of the constructs in a range of experiments we have designed assembly modules that encode the biotin ligases fused to different linkers as well as different commonly used subcellular localization sequences. We demonstrate the functionality of these vectors through biotinylation assays in tobacco (Nicotiana benthamiana) plants.
\end{abstract}




\section{INTRODUCTION}

Cellular processes are executed and controlled through interacting proteins. The characterization of protein-protein interactions (PPIs) is therefore a critical step in the understanding of biology. Conventional approaches such as affinity purification followed by mass spectrometry (AP-MS) or yeast-2-hybrid (Y2H) face a number of limitations including the isolation of interaction partners under non-physiological conditions following cell lysis or a dependency on high affinity interactions. In recent years, proximity-labelling has emerged as a powerful tool that allows for the detection of weak and transient interactions between proteins as well as the characterization of subcellular proteomes (reviewed in $[1,2]$ ).

One proximity labelling tool makes use of a promiscuous biotin ligase enzyme, termed BioID, which carries a point mutation in the biotin ligase BirA from Escherichia coli [3]. Expression of BioID fused to a bait protein in cells results in the biotinylation of proximal proteins which are either direct interactors or nearby proteins that do not directly interact with the fusion protein. As biotinylation is a rare occurrence in most organisms these biotinylated proteins can then be isolated by affinity purification using streptavidin coated beads and identified by mass spectrometry (MS) (Fig. 1A). Exogenous biotin is typically required for labelling of proteins so that labelling time is controlled by the addition of biotin to the cell, with labelling time generally between $15-18 \mathrm{~h}[3,4]$. Recently, novel variants of BioID have been developed through a directed evolution approach [5]. One of these mutant proteins, termed TurboID, displays a much faster rate of biotinylation in cells with labelling occurring within 10 min of biotin addition [5]. TurboID displays a higher level of activity at lower temperatures than BioID, possibly because BioID was derived from E. coli while TurboID was evolved in yeast [5]. In the same study researchers developed another highly active variant of this biotin ligase, termed MiniTurbo, which lacks the N-terminal (Nt) domain of TurboID resulting in a smaller variant (28 versus $35 \mathrm{kDa}$ ). MiniTurbo displays 
reduced stability $[4,5]$ but may produce a lower level of background biotinylation in the absence of exogenous biotin compared to TurboID [5]. The properties of these variants therefore allow for the capture of highly dynamic protein interactions at lower temperatures. To date TurboID has been shown to be active in a number of organisms and at a range of temperatures, including in Drosophila melanogaster, Caenorhabditis elegans, Arabidopsis thaliana, Nicotiana benthamiana and mammalian cells [5-8].

Over the last five decades a combination of type-II restriction enzymes and DNA ligases has been employed to generate recombinant plasmids. Although effective, this method can be time-consuming and may require multiple rounds of cloning steps. To overcome this limitation a number of cloning strategies have been developed in order to facilitate the rapid cloning of constructs. These include approaches making use of recombination-based cloning methods such as the Univector plasmid-fusion system, based on the Cre-lox system [9] or the widely used Gateway technology that makes use of the lambda phage DNA integration machinery $[10,11]$. Although the Gateway system allows for the efficient insertion of multiple genes in to a single vector and many Gateway compatible vectors now exist, there are a number of disadvantages to the system including the high cost of the enzymes required for the reactions and the generation of a $25 \mathrm{bp}$ 'scar' that is left flanking the integrated DNA as a result of the recombination site sequence. Another popular cloning strategy, termed the Golden Gate method, does not rely on recombination but instead makes use of type-IIS restriction enzymes [12]. These enzymes possess a specific DNA recognition site but cleave the DNA outside of this site, allowing for the generation of restriction site overhangs that can contain a customized sequence of nucleotides. Individual cloning modules are first generated as sequences flanked by two type-IIS restriction enzyme sites containing complementary overhangs with the desired preceding and succeeding modules. Upon digestion with the single type-IIS restriction enzyme the modules yield complementary overhangs allowing for 
their ligation in the presence of DNA ligase (Fig. 1B). With this method different combinations of individual modules can be quickly assembled together in a single reaction. Golden Gate cloning has been employed in a large number of studies and several resources exist including cloning kits such as the GoldenBraid $[13,14]$ and Golden GateWay series of vectors [15]. One cloning kit, termed GreenGate, was designed to facilitate rapid cloning of constructs using commonly used elements of plant transformation vectors [16]. Assembly modules are made up of six different types of element which typically include a promoter, Nterminal tag, coding sequence, $\mathrm{C}$-terminal tag, terminator and resistance sequence. Cleavage of these modules with the type-IIS restriction enzyme yields fragments with overhangs designated type A-G which are then ligated in to a backbone destination vector containing bacterial resistance and overhang type A-H, forming a higher order assembly of plasmid [16].

Here, we present a collection of constructs that have been designed for the rapid cloning of the TurboID and MiniTurbo biotin ligases using the Golden Gate cloning method which are compatible with the GreenGate series of vectors. To facilitate a range of experiments we have designed assembly modules that encode the biotin ligases fused to different linker-lengths as well as different commonly used localization sequences. To demonstrate the functionality of these vectors we have used elements of the collection to generate plant transformation vectors and transiently expressed them in tobacco plants (Nicotiana benthamiana). We demonstrate that the biotin-ligase fusion proteins encoded by these vectors localize to the expected subcellular compartment and can biotinylate proximal proteins in vivo. 
bioRxiv preprint doi: https://doi.org/10.1101/2021.10.15.464533; this version posted October $15,2021$. The copyright holder for this preprint (which was not certified by peer review) is the author/funder, who has granted bioRxiv a license to display the preprint in perpetuity. It is made available under aCC-BY 4.0 International license.

\section{Fig 1. Overview of biotin ligase proximity labelling and GreenGate cloning.}

(A) Activity of a promiscuous biotin ligase results in the generation and release of reactive bioAMP and the biotinylation of proximal proteins. Biotinylated proteins can be isolated by affinity purification using streptavidin coated beads. High stringency washes are performed to remove background. Following elution of proteins from the beads peptides can be identified by mass spectrometry.

(B) The Green Gate system of cloning utilizes a Golden Gate assembly approach. Entry vectors are first generated that contain single elements flanked by $B s a \mathrm{I}$ recognition sites and one of seven different overhangs (A-G). Upon digestion with $B s a \mathrm{I}$ individual elements are released from the entry vectors. Annealing of complementary overhangs and T4 ligase catalyzed DNA ligation results in the assembly of a final expression construct. Panel adapted from [16]. 


\section{RESULTS AND DISCUSSION}

\section{Generation of a biotin ligase cloning library}

In order to facilitate the rapid cloning of biotin ligase constructs we generated a library of 26 entry level cloning modules (Table 1). Each module is comprised of a TurboID or MiniTurbo coding sequence flanked by $B s a \mathrm{I}$ restriction enzyme sites. Modules were designed to allow for the enzyme to be positioned at the beginning, middle or end of a construct (overhangs B-

C / C-D/ D-E, respectively) following a promoter sequence, as per the Green Gate cloning kit layout [16] (see Materials and Methods).

The ability of biotin ligase to label an interactor is dependent on the proteins being proximal. In the case of a large protein, or a large protein complex, the labelling radius of a ligase fusion may not be sufficient to label interacting proteins [17]. The addition of flexible linker regions have previously been shown to increase the biotinylation range of a biotin ligase from its fused protein of interest [17]. Therefore, we included module constructs encoding each of the two ligases with an additional 28 aa long flexible linker region.

The inclusion of a biotin ligase-only control, whereby the biotin ligase is expressed without the protein of interest, is useful to interpret the potentially large list of peptides identified in a proximity labelling experiment [18]. This allows for the identification of proteins that may have been biotinylated because they are highly abundant in the cell or have some affinity to the biotin ligase protein itself. In order to identify physiologically relevant interaction partners this protein should be expressed at the same level as the biotin ligasegene of interest and should also be localized to the same subcellular compartment. To allow for some change in ligase localization we generated biotin ligase constructs encoding the ligases fused to a nuclear localization signal (NLS) derived from simian virus 40 (SV40) T antigen [19], a nuclear export signal (NES) from human immunodeficiency virus 1 (HIV-1) protein Rev [20] and an N-terminal signalling peptide from sweet potato sporamin targeting 
bioRxiv preprint doi: https://doi.org/10.1101/2021.10.15.464533; this version posted October 15,2021 . The copyright holder for this preprint (which was not certified by peer review) is the author/funder, who has granted bioRxiv a license to display the preprint in perpetuity. It is made available under aCC-BY 4.0 International license.

the protein to the endoplasmic reticulum (ER) [21]. To facilitate community use, all plasmids have been deposited with the Addgene repository (see Table 1; Materials and Methods).

\section{Table 1: A plasmid collection for proximity labelling.}

Plasmid names include the Green Gate terms ' $\mathrm{Nt}$ ', 'CDS' or ' $\mathrm{Ct}$ ' for different overhang pairs generated after plasmid digestion with $B s a \mathrm{I}$. Linker refers to flexible 28 aa long sequence, NLS to Nuclear Localization Sequence (SV40), NES to Nuclear Export Signal (HIV-1) and ER to Endoplasmic Reticulum targeting signal. pEntry vectors designated as ' $\mathrm{Ct}$ ' vectors (overhangs D-E) contain a stop codon at the end of the cassette. Vector maps are available at Addgene under the accession numbers provided.

\begin{tabular}{|c|c|c|c|c|}
\hline$\#$ & Name & Overhangs & Addgene \# & Description \\
\hline 1 & pEntry_TurboID_Nt & B - C & TurboID (cDNA) with different \\
overhangs.
\end{tabular}




\begin{tabular}{|c|c|c|c|}
\hline 11 & pEntry_TurboID_NES_CDS & $C-D$ & \multirow[t]{2}{*}{$\begin{array}{l}\text { Nuclear Export Signal (HIV-1) } \\
\text { with different overhangs. }\end{array}$} \\
\hline 12 & pEntry_TurboID_NES_Ct & $D-E$ & \\
\hline 13 & pEntry_ER_TurboID_Nt & $\mathrm{B}-\mathrm{C}$ & $\begin{array}{l}\text { Endoplasmic Reticulum } \\
\text { targeting signal fused to } \\
\text { TurboID (cDNA). }\end{array}$ \\
\hline 14 & pEntry_MiniTurbo_Nt & $\mathrm{B}-\mathrm{C}$ & \multirow{3}{*}{$\begin{array}{l}\text { MiniTurbo (cDNA) with } \\
\text { different overhangs. }\end{array}$} \\
\hline 15 & pEntry_MiniTurbo_CDS & $C-D$ & \\
\hline 16 & pEntry_MiniTurbo_Ct & $D-E$ & \\
\hline 17 & pEntry_MiniTurbo_linker_Nt & $\mathrm{B}-\mathrm{C}$ & \multirow{3}{*}{$\begin{array}{c}\text { MiniTurbo (cDNA) fused to } 28 \\
\text { aa linker region with different } \\
\text { overhangs. }\end{array}$} \\
\hline 18 & pEntry_MiniTurbo_linker_CDS & $\mathrm{C}-\mathrm{D}$ & \\
\hline 19 & pEntry_Linker_MiniTurbo_Ct & $D-E$ & \\
\hline 20 & pEntry_MiniTurbo_NLS_Nt & $\mathrm{B}-\mathrm{C}$ & \multirow{3}{*}{$\begin{array}{l}\text { MiniTurbo (cDNA) fused to } \\
\text { Nuclear Localization Signal } \\
\text { (SV40) with different } \\
\text { overhangs. }\end{array}$} \\
\hline 21 & pEntry_MiniTurbo_NLS_CDS & $C-D$ & \\
\hline 22 & pEntry_MiniTurbo_NLS_Ct & $D-E$ & \\
\hline 23 & pEntry_MiniTurbo_NES_Nt & $\mathrm{B}-\mathrm{C}$ & \multirow{3}{*}{$\begin{array}{l}\text { MiniTurbo (cDNA) fused to } \\
\text { Nuclear Export Signal (HIV-1) } \\
\text { with different overhangs. }\end{array}$} \\
\hline 24 & pEntry_MiniTurbo_NES_CDS & $C-D$ & \\
\hline 25 & pEntry_MiniTurbo_NES_Ct & $D-E$ & \\
\hline 26 & pEntry_ERtarget_MiniTurbo_Nt & $\mathrm{B}-\mathrm{C}$ & $\begin{array}{l}\text { Endoplasmic Reticulum } \\
\text { targeting signal fused to } \\
\text { MiniTurbo (cDNA). }\end{array}$ \\
\hline
\end{tabular}




\section{TurboID fusion proteins are active in tobacco leaves and localize to different subcellular compartments}

To ensure that the different localization signals do not interfere with biotin ligase activity and that they drive localization of the protein to the predicted cellular compartment we generated, using the entry modules described above, constructs expressing TurboID under the UBQ10 promoter (UBQ10p) fused to Green Fluorescent Protein (GFP) and encoding one of the localization signals (see Fig. 2A; Materials and Methods). These constructs were then transformed into tobacco leaf cells by Agrobacterium-infiltration. Using immuno-blotting we found that the expression of TurboID fusion proteins led to higher levels of biotinylated proteins in total protein extracts when compared to untransformed plants, likely as a consequence of biotin being naturally present in plant cells [6]. The signal for biotinylated proteins increased upon addition of extracellular biotin in to the leaf, consistent with previously described TurboID experiments in tobacco (Fig. 2B) [6].

To observe the localization of the different TurboID-GFP fusion proteins we took sections of tobacco leaves transiently expressing the constructs and examined their fluorescence pattern using confocal microscopy. TurboID fused with GFP and the different localization signals gave fluorescent signals consistent with their targeted subcellular localization to the nucleus, cytoplasm and endoplasmic reticulum when compared with non-transformed leaves (Fig. 3). Taken together these results indicate that TurboID constructs generated with the library entry modules are active in planta and allow for the efficient targeting of TurboID protein fusions to different subcellular compartments. 


\section{Fig 2. TurboID fusion proteins are active in tobacco leaves.}

(A) Schematic representation of the TurboID-GFP fusion proteins expressed in tobacco leaves.

(B) Expression of the different TurboID-GFP fusion proteins in tobacco leaves increases the abundance of biotinylated proteins. Total protein extracts were prepared from nontrnasformed $N$. benthamiana leaves as well as from leaves transiently expressing the TurboID-GFP fusion proteins. TurboID fusion proteins were detected by immunoblotting using anti-GFP antibodies while biotinylated proteins were detected using streptavidin-HRP. TurboID-GFP-NLS/NES and ER-TurboID-GFP are expected to migrate at $\sim 64 \mathrm{kDa}$ and 66 $\mathrm{kDa}$, respectively. Experiment was conducted twice with similar results.

\section{Fig 3. Subcellular localization of TurboID-GFP fusion proteins.}

Confocal microscopy images of abaxial epidermal cells from $N$. benthamiana leaves $3 \mathrm{~d}$ after infiltration with A. tumefaciens containing TurboID-GFP (TID) constructs with (A) a Nuclear-Localization Signals (NLS), (B) a Nuclear-Export Signal (NES), or (C) an Endoplasmic Reticulum targeting signal (ER). In (D), abaxial epidermal cells from nontransformed leaves are shown. Scale bars: $20 \mu \mathrm{m}$. 


\section{TurboID fusion proteins efficiently label interaction partners}

We next tested if a protein-TurboID fusion construct generated with the entry modules resulted in the labelling of physiologically relevant interaction partners. To this end we performed a co-expression experiment in tobacco leaves using two Arabidopsis MADS domain transcription factors, APETALA3 (AP3) and PISTILLATA (PI), which are known to physically interact with one another and function as an obligate heterodimer during flower development $[22,23]$. Tobacco leaves co-expressing an AP3-GFP construct with a PITurboID fusion protein (PI-TurboID-3xMyc) (Fig. 4A) were infiltrated with either a mock or biotin-containing solution and tissue was collected after 3 hours. As AP3 and PI colocalize to the nucleus we generated nuclear protein extracts (see Materials and Methods). After immunoprecipitation of AP3-GFP using anti-GFP antibody coated beads a band at approximately $55 \mathrm{kDa}$ could be observed corresponding to biotinylated AP3-GFP (Fig. 4B). This band was strongest in biotin-treated leaves but was also present in leaves not treated with exogenous biotin (Fig. 4B). This suggests that endogenous biotin in tobacco leaves is sufficient for some labelling of proximal proteins, albeit to a lesser extent. Another band, which migrated at the same size as PI-TurboID-3xMyc $(\sim 65 \mathrm{kDa})$, was observed on the membrane (Fig. 4B). This is likely auto-biotinylated PI-TurboID-3xMyc which coimmunoprecipitated with AP3-GFP, consistent with previous reports showing biotin ligase auto-biotinylation $[3,24]$. These results demonstrate that the entry modules generated for TurboID cloning can be used to construct a TurboID fusion protein that labels physiologically relevant interaction partners in vivo. 
bioRxiv preprint doi: https://doi.org/10.1101/2021.10.15.464533; this version posted October 15,2021 . The copyright holder for this preprint (which was not certified by peer review) is the author/funder, who has granted bioRxiv a license to display the preprint in perpetuity. It is made available under aCC-BY 4.0 International license.

\section{Fig 4. TurboID expression construct labels interaction partners of bait protein.}

(A) Schematic representation of the fusion proteins expressing the known interactors AP3 and PI fused to GFP and TurboID-3xMyc, respectively, expressed in tobacco leaves.

(B) Biotinylation of AP3-GFP by PI-TurboID-Myc. Nuclear protein extracts were prepared from non-transformed $N$. benthamiana leaves or from leaves transiently expressing fusion the proteins. Immunoprecipitates were obtained using anti-GFP coated beads (ChromoTek). AP3-GFP, PI-TurboID-Myc and biotinylated proteins were detected using anti-GFP and antiMyc antibodies and streptavidin-HRP, respectively. AP3-GFP is predicted to migrate at $\sim 55$ $\mathrm{kDa}$, PI-TurboID-Myc at $\sim 65 \mathrm{kDa}$. This experiment was conducted twice using different antiGFP coated beads (ChromoTek GFP-Trap or Miltenyi $\mu$ MACS Magnetic beads) with similar results. In the upper-most panel, a longer exposure ( $5 \mathrm{~min}$ ) was chosen to better visualize faint bands appearing in the absence of exogenous biotin treatment in an extract from leaves expressing both the AP3-GFP and PI-TurboID-Myc fusion proteins. 


\section{MATERIALS AND METHODS}

\section{Plant growth conditions}

$N$. benthamiana plants were grown on a medium consisting of compost, perlite and vermiculite in a ratio of $3: 1: 1$. The plants were grown under constant illumination at $20^{\circ} \mathrm{C}$ after being incubated at $4^{\circ} \mathrm{C}$ in the dark for $5 \mathrm{~d}$.

\section{Generation of biotin ligase cloning modules}

All cloning procedures are described in the Supplementary Materials. Oligonucleotides used and plasmids generated are described in Table S1 and S2, respectively.

\section{Transient gene expression in $N$. benthamiana}

Agrobacterium tumefaciens cells (C58 pGV2260 containing pSOUP) were transformed with plasmid DNA and plated on LB plates supplemented with appropriate antibiotics for 2-3 $d$ at $28^{\circ} \mathrm{C}$. A colony from this plate was used to inoculate LB medium supplemented with appropriate antibiotics and grown overnight at $28^{\circ} \mathrm{C}$ with shaking $(\sim 225 \mathrm{rpm})$. A. tumefaciens cells were centrifuged at $4,000 \mathrm{~g}$ for $10 \mathrm{~min}$ and resuspended at an $\mathrm{OD}_{600}$ of 0.75 in Infiltration Medium (10 mM MES pH 5.6, $10 \mathrm{mM} \mathrm{MgCl}_{2}, 150 \mu \mathrm{M}$ acetosyringone). Approximately 4-5 week old $N$. benthamiana leaves were infiltrated with this solution using a blunt $1 \mathrm{~mL}$ syringe and leaf tissue was examined or stored in $\mathrm{N}_{2}(1)$ after $3 \mathrm{~d}$.

\section{Protein extraction and immunoblotting}

To prepare total protein extracts from $N$. benthamiana leaves for immunoblotting, plant tissue was ground to a fine powder in $\mathrm{N}_{2}(1)$. This powder was then resuspended in standard $2 \mathrm{X}$ SDS-loading buffer and boiled for $5 \mathrm{~min}\left(95^{\circ} \mathrm{C}\right)$. Samples were centrifuged and the supernatants were used for SDS-PAGE and immunoblotting. Antibodies were diluted in 1X phosphate-buffered saline plus Tween $20(0.05 \%)$ with either $5 \%(\mathrm{w} / \mathrm{v})$ milk powder (for 
anti-GFP and anti-Myc immunoblots) or 5\% Bovine Serum Albumin (BSA) powder (streptavidin-HRP immunoblots). Primary antibodies used were: anti-GFP [1:5,000], Roche \#1181446001; anti-Myc [1:1,000], \#M5546, Sigma; streptavidin-HRP [0.2 $\mu \mathrm{g} / \mathrm{ml}]$, Thermo Fisher Scientific \#S911.

\section{Confocal imaging to visualize TurboID-GFP subcellular localization}

In order to visualize GFP-tagged proteins expressed in tobacco leaves a Zeiss LSM 710 laser scanning confocal microscope was used. Tobacco leaf tissue was placed on a slide with $\mathrm{H}_{2} \mathrm{O}$ to prevent excessive drying and covered with a coverslip. Protein localization was visualized from the abaxial leaf side using a $488 \mathrm{~nm}$ excitation wavelength. Image processing was performed in FIJI (Image J) [25].

\section{N. benthamiana nuclear enrichment and AP3-GFP immunoprecipitation}

Three days after Agrobacterium-infiltration of tobacco leaves (as described above) leaf tissue was infiltrated with a biotin solution $\left(250 \mu \mathrm{M}\right.$ biotin, $\left.10 \mathrm{mM} \mathrm{MgCl}_{2}\right)$ or a mock solution (10 $\mathrm{mM} \mathrm{MgCl} 2$ ) using a blunt $1 \mathrm{~mL}$ syringe. After $3 \mathrm{~h}$ of labelling leaves were frozen in $\mathrm{N}_{2}(\mathrm{l})$ and ground to a fine powder. The powder was resuspended in $30 \mathrm{~mL}$ buffer M1 (10 mM sodium phosphate, $\mathrm{pH}$ 7, $0.1 \mathrm{M} \mathrm{NaCl}, 1 \mathrm{M}$ 2-methyl 2,4-pentanediol, $10 \mathrm{mM} \beta$-mercaptoethanol and 1X Complete Protease Inhibitor Cocktail (CPIC, Sigma \#1169749800). The solution was left at $4{ }^{\circ} \mathrm{C}$ on a rotator for $10 \mathrm{~min}$ before being filtered twice through Miracloth (Merck Millipore) and spun at $1,000 \mathrm{~g}$ for $20 \mathrm{~min}$ at $4^{\circ} \mathrm{C}$. The pellet was gently resuspended in $5 \mathrm{~mL}$ ice-cold M2 buffer (M1 buffer with additional $10 \mathrm{mM} \mathrm{MgCl}_{2}$ and $0.6 \%$ Triton X-100) before being centrifuged at $1,000 \mathrm{~g}$ for $10 \mathrm{~min}$ at $4^{\circ} \mathrm{C}$. Pellet washes with $\mathrm{M} 2$ buffer were repeated 3 times. The pellet was then gently resuspended in $1 \mathrm{~mL} \mathrm{M} 3$ buffer $(10 \mathrm{mM}$ sodium phosphate, $\mathrm{pH} 7,0.1 \mathrm{M} \mathrm{NaCl}, 10 \mathrm{mM} \beta$-mercaptoethanol and $1 \mathrm{X}$ CPIC) and centrifuged at 1,000 $\mathrm{g}$ for $10 \mathrm{~min}$ at $4^{\circ} \mathrm{C}$. The pellet was resuspended in $300 \mu \mathrm{L}$ lysis buffer $(150 \mathrm{mM} \mathrm{NaCl}, 1 \%$ Triton 
$\mathrm{X}-100,50 \mathrm{mM}$ Tris $\mathrm{HCl} \mathrm{pH}$ 8.0, 0.1\% SDS, 0.5\% sodium deoxycholate) before being sonicated using a Diagenode Bioruptor Pico for 10 cycles of $30 \mathrm{~s}$ on/off at $4^{\circ} \mathrm{C}$, and centrifuged at $12,000 \mathrm{x} g$ for $5 \mathrm{~min}$ at $4^{\circ} \mathrm{C}$. Protein concentration was determined using an Amido Black assay[26]. Protein extracts were diluted to $800 \mu \mathrm{L}$ in IP buffer $(150 \mathrm{mM} \mathrm{NaCl}$, 1\% Triton $\mathrm{X}-100,50 \mathrm{mM}$ Tris $\mathrm{HCl} \mathrm{pH} 8.0)$. Anti-GFP coated beads were added $(25 \mu \mathrm{L}$ ChromoTek GFP-Trap or $50 \mu \mathrm{L}$ MACS magnetic) and IP was carried out as per the manufacturers' instructions. Proteins were eluted from the beads by addition of 2X SDSloading buffer and incubation for $5 \mathrm{~min}$ at $95^{\circ} \mathrm{C}$. The eluate was stored at $-20^{\circ} \mathrm{C}$ until being analyzed by immunoblotting.

\section{Availability of the plasmid vectors}

All constructs for proximity labelling have been deposited with Addgene under accession numbers XXX (see Table 1) and are available from there.

\section{Acknowledgements}

We thank Dr. Brendan Davies for discussions and Dr. Emmanuelle Graciet for help with experiments. We also thank Drs. Chris Greene and Matthew Campbell for help with confocal microscopy. Funding for this work was provided by Science Foundation Ireland and the Environmental Protection Agency (grant 16/IA/4559 to F.W.). 


\section{REFERENCES}

1. Kim DI, Roux KJ. Filling the Void: Proximity-Based Labeling of Proteins in Living Cells. Trends Cell Biol. 2016;26(11):804-17.

2. Trinkle-Mulcahy L. Recent advances in proximity-based labeling methods for interactome mapping. F1000Res. 2019;8.

3. Roux KJ, Kim DI, Raida M, Burke B. A promiscuous biotin ligase fusion protein identifies proximal and interacting proteins in mammalian cells. J Cell Biol. 2012;196(6):801-10.

4. May DG, Scott KL, Campos AR, Roux KJ. Comparative Application of BioID and TurboID for Protein-Proximity Biotinylation. Cells. 2020;9(5).

5. Branon TC, Bosch JA, Sanchez AD, Udeshi ND, Svinkina T, Carr SA, et al. Efficient proximity labeling in living cells and organisms with TurboID. Nat Biotechnol. 2018;36(9):880-7.

6. Mair A, Xu SL, Branon TC, Ting AY, Bergmann DC. Proximity labeling of protein complexes and cell-type-specific organellar proteomes in. Elife. 2019;8.

7. Arora D, Abel NB, Liu C, Van Damme P, Yperman K, Eeckhout D, et al. Establishment of Proximity-Dependent Biotinylation Approaches in Different Plant Model Systems. Plant Cell. 2020;32(11):3388-407.

8. Cho KF, Branon TC, Udeshi ND, Myers SA, Carr SA, Ting AY. Proximity labeling in mammalian cells with TurboID and split-TurboID. Nat Protoc. 2020;15(12):3971-99.

9. Liu Q, Li MZ, Leibham D, Cortez D, Elledge SJ. The univector plasmid-fusion system, a method for rapid construction of recombinant DNA without restriction enzymes. Curr Biol. 1998;8(24):1300-9.

10. Hartley JL, Temple GF, Brasch MA. DNA cloning using in vitro site-specific recombination. Genome Res. 2000;10(11):1788-95.

11. Sasaki Y, Sone T, Yoshida S, Yahata K, Hotta J, Chesnut JD, et al. Evidence for high specificity and efficiency of multiple recombination signals in mixed DNA cloning by the Multisite Gateway system. J Biotechnol. 2004;107(3):233-43.

12. Engler C, Kandzia R, Marillonnet S. A one pot, one step, precision cloning method with high throughput capability. PLoS One. 2008;3(11):e3647.

13. Sarrion-Perdigones A, Vazquez-Vilar M, Palací J, Castelijns B, Forment J, Ziarsolo P, et al. GoldenBraid 2.0: a comprehensive DNA assembly framework for plant synthetic biology. Plant Physiol. 2013;162(3):1618-31. 
14. Vazquez-Vilar M, Bernabé-Orts JM, Fernandez-Del-Carmen A, Ziarsolo P, Blanca J, Granell A, et al. A modular toolbox for gRNA-Cas9 genome engineering in plants based on the GoldenBraid standard. Plant Methods. 2016;12:10.

15. Kirchmaier S, Lust K, Wittbrodt J. Golden GATEway cloning--a combinatorial approach to generate fusion and recombination constructs. PLoS One. 2013;8(10):e76117.

16. Lampropoulos A, Sutikovic Z, Wenzl C, Maegele I, Lohmann JU, Forner J. GreenGate--a novel, versatile, and efficient cloning system for plant transgenesis. PLoS One.

2013;8(12):e83043.

17. Kim DI, Jensen SC, Noble KA, Kc B, Roux KH, Motamedchaboki K, et al. An improved smaller biotin ligase for BioID proximity labeling. Mol Biol Cell. 2016;27(8):1188-96.

18. Roux KJ, Kim DI, Burke B, May DG. BioID: A Screen for Protein-Protein Interactions. Curr Protoc Protein Sci. 2018;91:19.23.1-19.23.15.

19. Collas P, Aleström P. Nuclear localization signal of SV40 T antigen directs import of plasmid DNA into sea urchin male pronuclei in vitro. Mol Reprod Dev. 1996;45(4):431-8.

20. Fischer U, Huber J, Boelens WC, Mattaj IW, Lührmann R. The HIV-1 Rev activation domain is a nuclear export signal that accesses an export pathway used by specific cellular RNAs. Cell. 1995;82(3):475-83.

21. Koide Y, Matsuoka K, Ohto M, Nakamura K. The N-terminal propeptide and the C terminus of the precursor to 20-kilo-dalton potato tuber protein can function as different types of vacuolar sorting signals. Plant Cell Physiol. 1999;40(11):1152-9.

22. Tröbner W, Ramirez L, Motte P, Hue I, Huijser P, Lönnig WE, et al. GLOBOSA: a homeotic gene which interacts with DEFICIENS in the control of Antirrhinum floral organogenesis. EMBO J. 1992;11(13):4693-704.

23. Riechmann JL, Krizek BA, Meyerowitz EM. Dimerization specificity of Arabidopsis MADS domain homeotic proteins APETALA1, APETALA3, PISTILLATA, and AGAMOUS. Proc Natl Acad Sci U S A. 1996;93(10):4793-8.

24. Zhang Y, Song G, Lal NK, Nagalakshmi U, Li Y, Zheng W, et al. TurboID-based proximity labeling reveals that UBR7 is a regulator of N NLR immune receptor-mediated immunity. Nat Commun. 2019;10(1):3252.

25. Schindelin J, Arganda-Carreras I, Frise E, Kaynig V, Longair M, Pietzsch T, et al. Fiji: an open-source platform for biological-image analysis. Nat Methods. 2012;9(7):676-82.

26. Popov N, Schmitt M, Schulzeck S, Matthies H. [Reliable micromethod for determination of the protein content in tissue homogenates]. Acta Biol Med Ger. 1975;34(9):1441-6. 


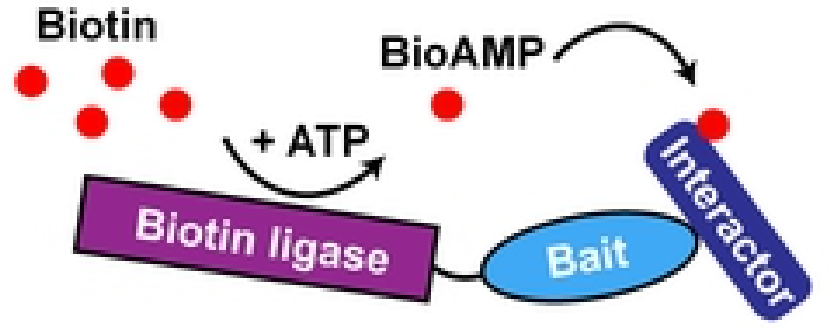

Streptavidin coated beads

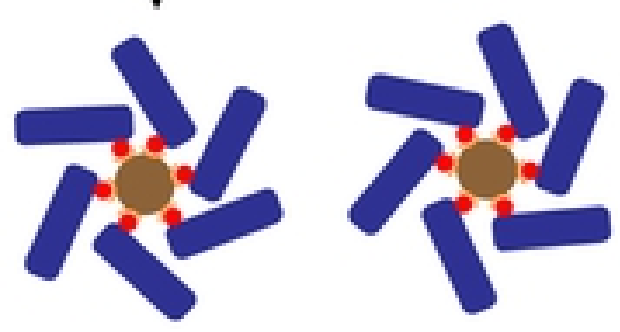

High stringency washes

Elution

Preparation for MS analysis

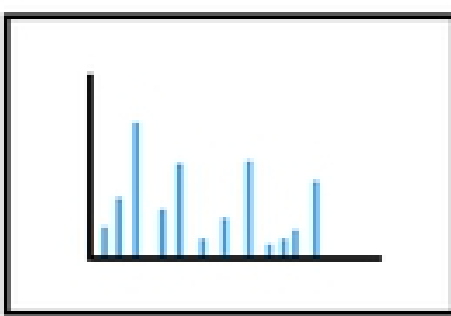

MS analysis
B

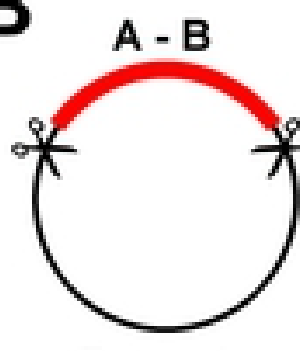

Promoter

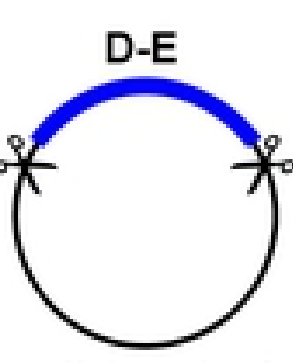

Terminator

Selection

+ Bsal restriction enzyme

+ DNA ligase

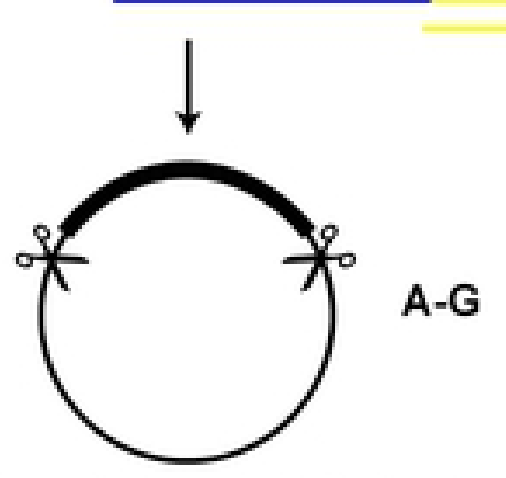

Destination vector (Bacterial ori, resistance)

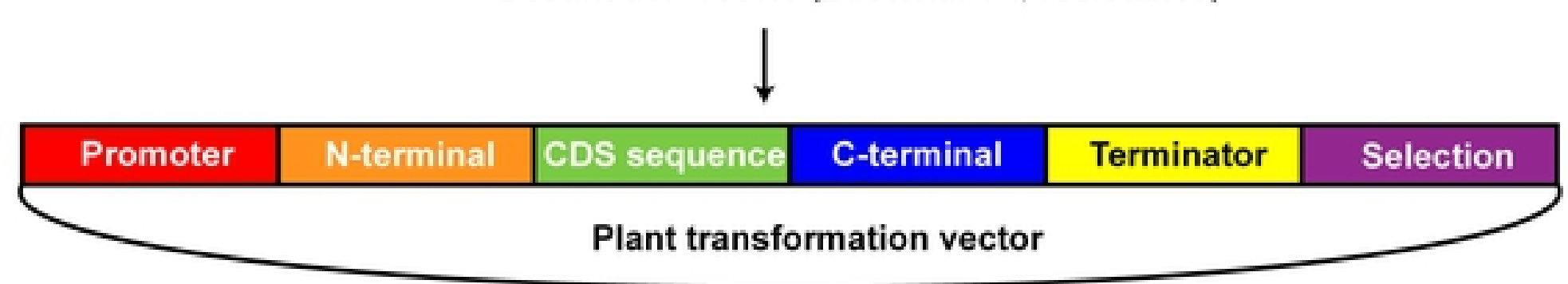

Fig 1. 
GFP

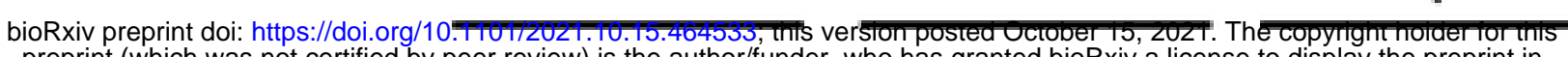

$$
\text { Biotin }-+\infty-++
$$

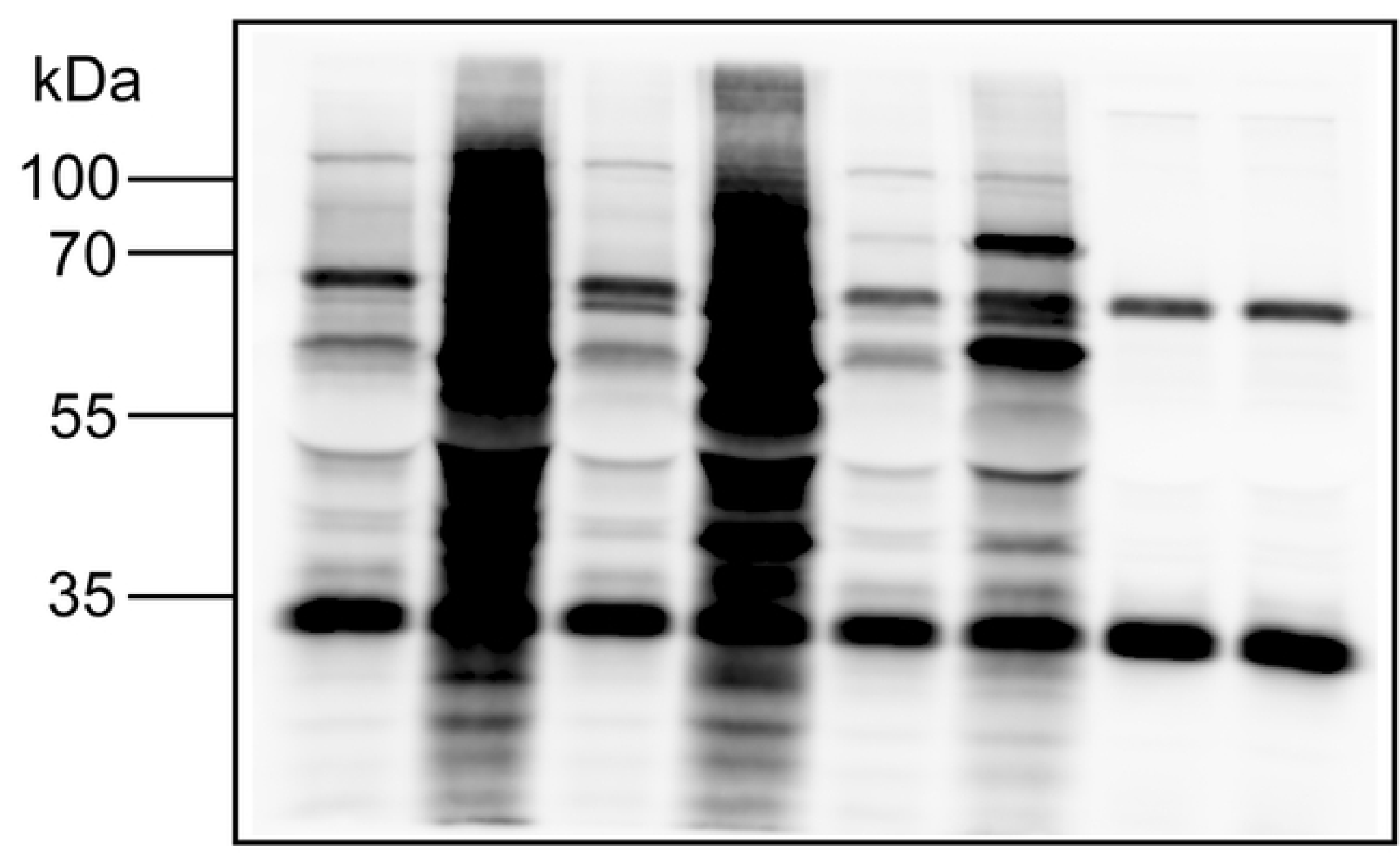

\section{Streptavidin-HRP}
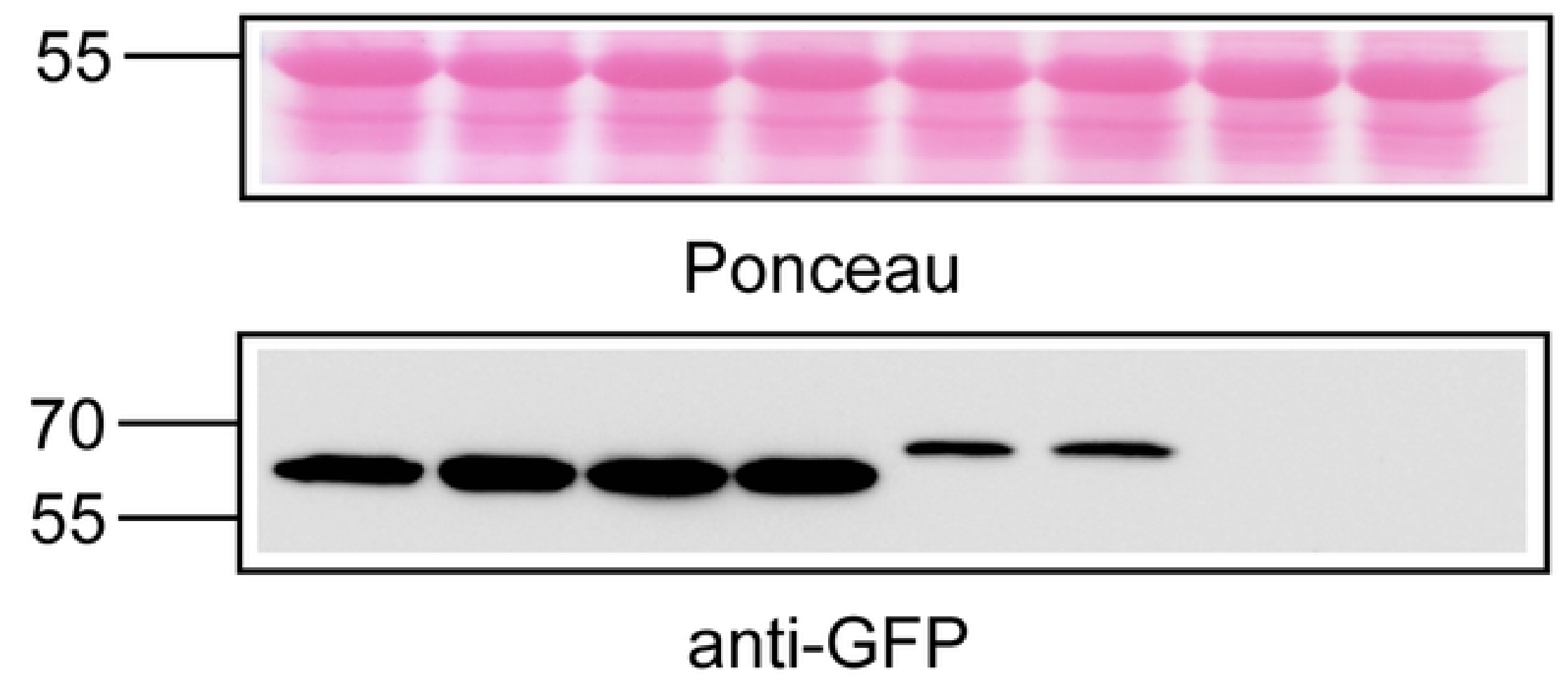

Fig 2. 
A UBQ10p

\begin{tabular}{l|l} 
AP3 & GFP
\end{tabular}

UBQ10p

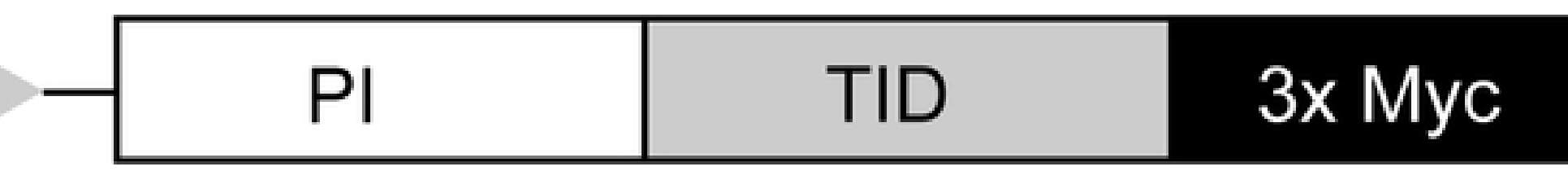

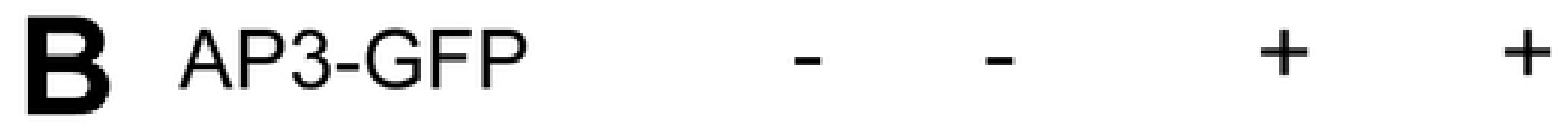

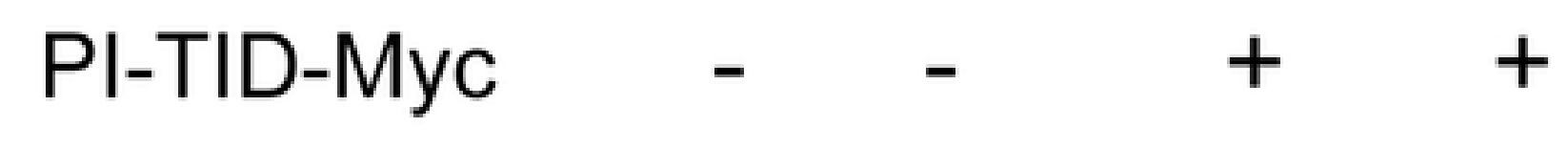

Biotin $\quad-\quad+\quad+\quad-\quad+$

$\mathrm{kDa}$
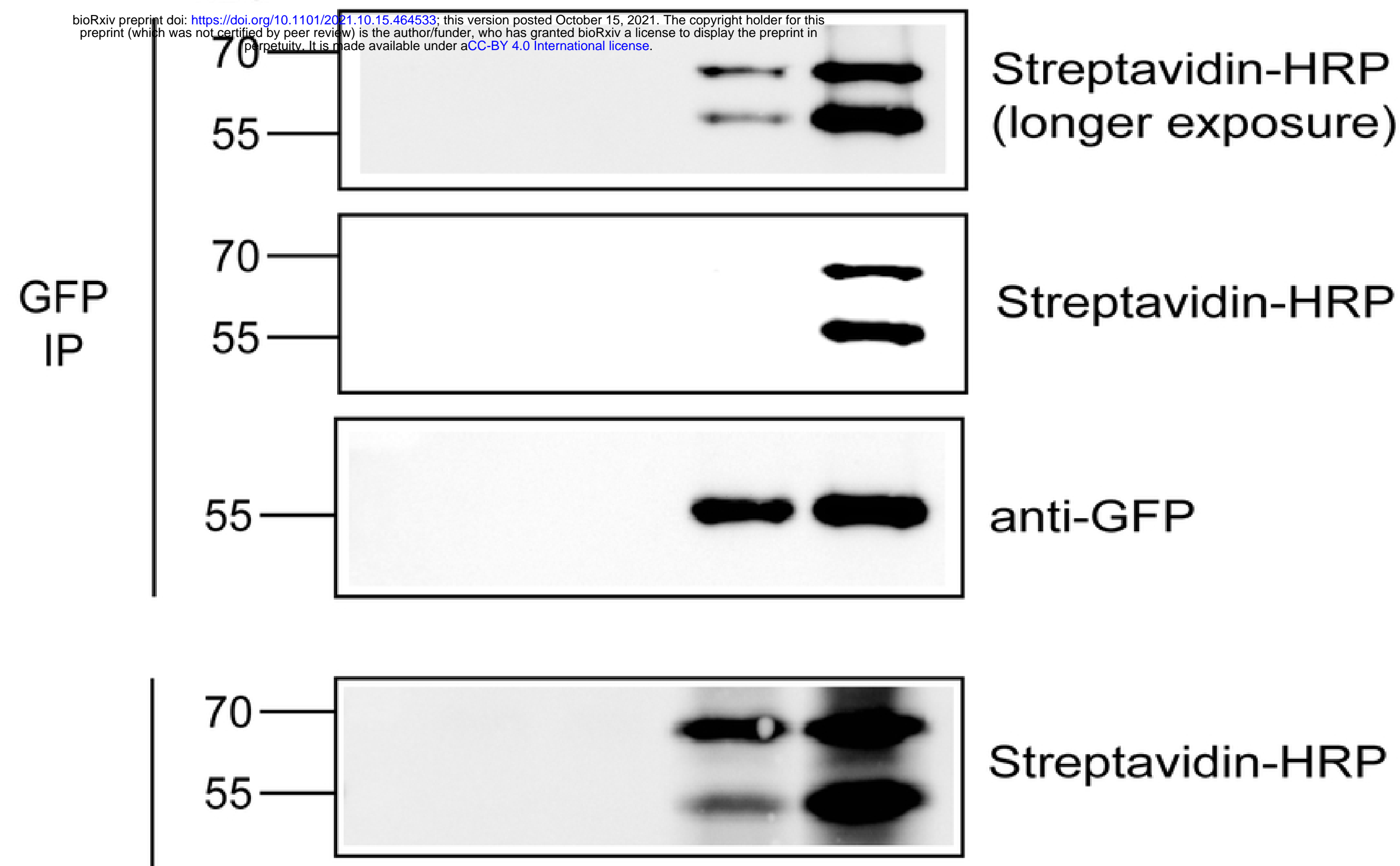

Streptavidin-HRP

Input

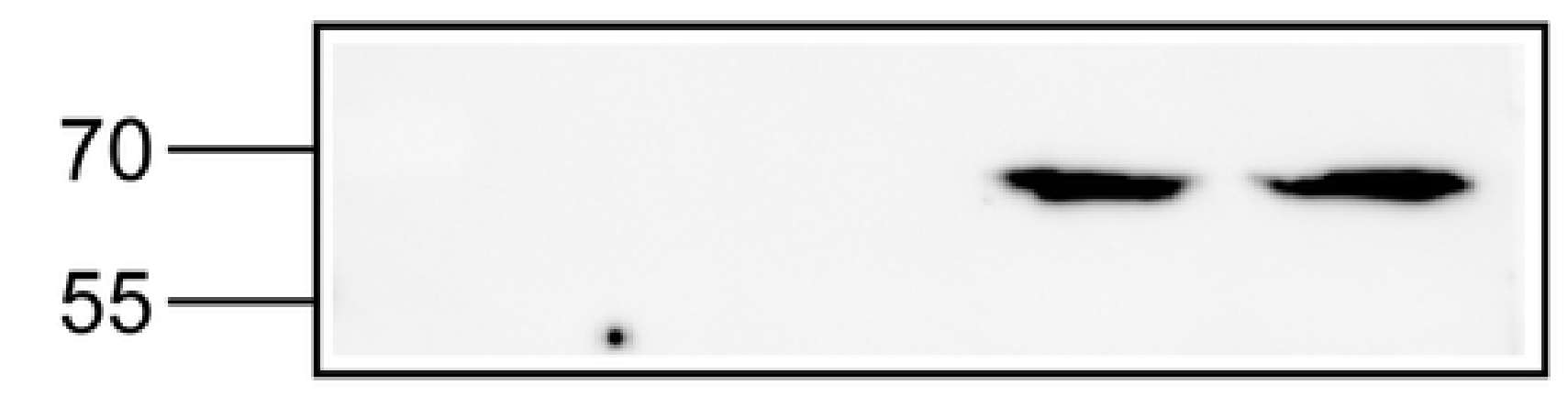

anti-Myc

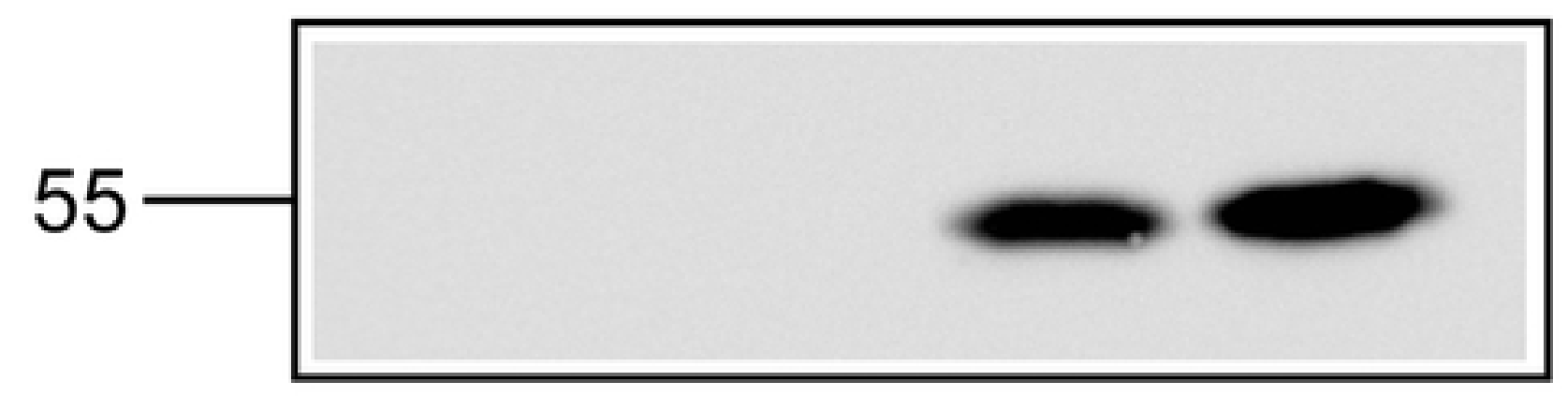

anti-GFP

Fig 4. 